Komet/ Gebr. Brasseler

\title{
Starker Support beim Einstieg
}

In der Fülle von Optionen sehnt sich jeder beim Schritt in die Selbstständigkeit nach Sicherheit. Frisch von der Uni die eigene Praxis einrichten da überkommt den Zahnarzt schnell die Qual der Wahl! Dieser Beitrag fasst zusammen, wie Komet dem Praxisgründer beim Start zur Seite steht und welche Qualitäten und Hilfestellungen er jetzt und in Zukunft von dem deutschen Traditionsunternehmen erwarten darf.

Speziell beim Einstieg in die Selbstständigkeit ist es wichtig, mit Instrumenten zu arbeiten, die sicher und logisch aufgebaut sind. Gebr. Brasseler bietet die größte Produktpalette an rotierenden Instrumenten deutschlandweit. Selbsterklärende Nummerierungen an den Instrumenten leisten immer elegante Hilfe, um Arbeitsabläufe darzu- stellen und um speziell am Anfang Sicherheit zu bieten.

Vielleicht darf man die Instrumente auch einfach als schlau bezeichnen, so wie z.B. das Experten-Set 4562 zur Präparation keramischer Inlays und Teilkronen. Die zehn ausgewählten Instrumente sind perfekt aufeinander abgestimmt und drei Schleifkörper wurden mit einer speziell berechneten Tiefenmarkierung versehen. Dieses kleine optische Detail vereinfacht und systematisiert die Kavitätengestaltung - ein wesentlicher Beitrag zur Qualitätssicherung!

Das zweite Produkt EasyShape steht beispielhaft für eine sichere Endodontie. Mit dem Feilensystem kann der Wurzelkanal maschinell auf ganzer Länge effizient aufbereitet werden, denn das Schneiden- design garantiert Sicherheit und eine stufenlose Vorgehensweise - ideal für den Einsteiger in dieser diffizilen Disziplin!

Wer Komet-Produkte kauft, muss nicht den Umweg über den Dentalhandel gehen. Ihr Fachberater betreut Sie individuell mit viel Know-how, damit sich Ihr neues Praxiskonzept und Ihre Bedürfnisse in den Komet-Instrumenten widerspiegeln.

Die Vorteile mit Komet als Partner bei der Praxisgründung liegen offensichtlich nicht nur auf, sondern in der Hand. Wir freuen uns auf die Zusammenarbeit!

ス Tel. 05261 - 701-700

info@brasseler.de

www.kometdental.de

\section{M ESPE}

\section{Espertise Talent Award 2010}

Um hochbegabte zahnmedizinische Nachwuchswissenschaftler zu fördern, veranstaltet 3M ESPE alle zwei Jahre den Espertise Talent Award. Der Gewinner sichert sich ein Stipendium für einen dreimonatigen Studienaufenthalt an einer Universität im Ausland im Wert von mehr als 10.000,Euro. Wer dieses Karrieresprungbrett nutzen möchte, kann sich bis zum 4. August 2010 zur Teilnahme anmelden. Ermittelt wird der beste deutschsprachige, wissenschaftliche Nachwuchsreferent 2010 am 16. und 17. September 2010 in Seefeld anhand eines Kurzvortrags über eigene Forschungsarbeiten.
„Der intensive Dialog mit Universitäten in der ganzen Welt hat bei uns bereits Tradition und mit dem Talent Award wollen wir einen besonderen Beitrag zur Förderung des wissenschaftlichen Nachwuchses und der internationalen Kooperation leisten", erklärt Dr. Gerhard Kultermann, Head of Customer Information Center CIC in Seefeld. Teilnahmeberechtigt sind approbierte Zahnärzte und Materialwissenschaftler, die am Beginn ihrer wissenschaftlichen Karriere stehen und bereits erste Erfahrungen in Forschung und Lehre gesammelt haben.
Das Anmeldeformular kann unter www.3mespe.de/uni in der Rubrik Espertise Talent Award abgerufen werden und kann bis spätestens zum 4. August 2010 per Post, Fax oder Mail ein-: gereicht werden.

\section{入 $3 \mathrm{M}$ ESPE AG}

Mellanie Schopp

ESPE Platz | 82229 Seefeld

Fax +49 (0) 8152/ 700-2675

mellanie.schopp@mmm.com
Ivoclar Vivadent

\section{i-Kids Programm}

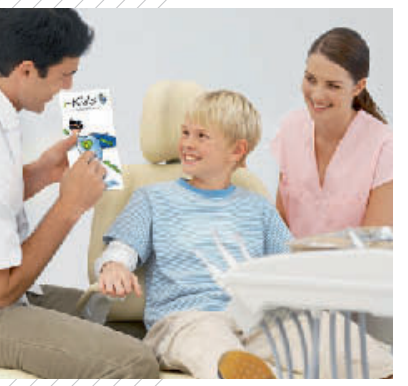

Mit dem i-Kids-Programm nimmt sich Ivoclar Vivadent der besonderen Bedürfnisse bei der zahnärztlichen Betreuung junger Menschen an. Das Programm umfasst Qualitätsprodukte und Serviceleistungen zur effektiven und effizienten Umsetzung von Behandlungskonzepten bei Kindern und Jugendlichen. Die i-Kids-Produkte decken ein weites Spektrum ab: Risikoanalyse und Diagnostik, Prävention, minimal-invasive Therapie oder Restauration sowie Pflege und Nachsorge. Dabei ermöglichen die aufeinander abgestimmten Produkte ein differenziertes Mundgesundheits-Management: Zur Wahl stehen Diagnostika, Therapeutika, Restaurationsmaterialien sowie Pflegeprodukte. Der Einsatz aller Produkte ist in internationalen Studien dokumentiert und im Praxisalltag klinisch erprobt.
Doch bei dem Programm geht es um weit mehr: i-Kids will eine positive Erlebniswelt in der Praxis schaffen. So gibt es bereits

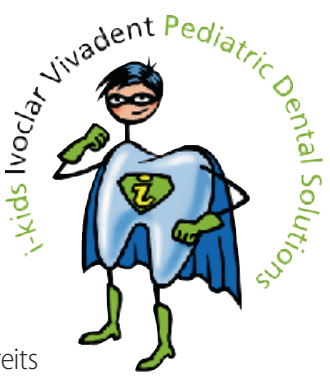
Tapferkeitsurkunden, Poster, Taschen und Stempel aus dem i-Kids-Programm.

$\boldsymbol{\lambda}$ Tel. 07961 - 889-0 info@ivoclarvivadent.de www.ivoclarvivadent.de 